\title{
Optimal timing of regime switching in optimal growth models: A Sobolev space approach
}

\author{
Erol Dogan ${ }^{\mathrm{a}}$, Cuong Le Van ${ }^{\mathrm{b}, \mathrm{c}}$, Cagri Saglam ${ }^{\mathrm{a}, *}$ \\ ${ }^{a}$ Department of Economics, Bilkent University, Turkey \\ ${ }^{\mathrm{b}}$ CNRS, Paris School of Economics, France \\ ${ }^{\mathrm{c}}$ Exeter University Business School, United Kingdom
}

\section{A R T I C L E I N F O}

\section{Article history:}

Received 20 July 2009

Received in revised form

20 November 2010

Accepted 22 November 2010

Available online 5 December 2010

\section{JEL classification:}

C61

041

Keywords:

Multi-stage optimal control

Sobolev spaces

Optimal growth models

\begin{abstract}
A B S T R A C T
This paper analyses the optimal timing of switching between alternative and consecutive regimes in optimal growth models. We derive the appropriate necessary conditions for such problems by means of standard techniques from the calculus of variations and some basic properties of Sobolev spaces.
\end{abstract}

(c) 2010 Elsevier B.V. All rights reserved.

\section{Introduction}

Many decision processes arising in economics involve a finite number of discrete changes both in the structure of the system and the objective functional over the course of the planning horizon. This paper presents the necessary conditions for the optimal timing of switches between these alternative regimes which are of particular importance.

Some early contributions to the optimal regime switching problems have proposed multi-stage optimal control techniques that recall the Pontryagin maximum principle from a dynamic programming perspective (see Tomiyama, 1985; Tomiyama and Rossana, 1989; Makris, 2001; Saglam, 2010). The main idea is to reduce a two-stage problem into a standard one with a dynamic programming approach, first by solving the post-switch problem and then attaching its value function to the pre-switch one with the Pontryagin maximum principle concluding at the intermediate steps. The illustrations of this technique on technology adoption problems can be found in Boucekkine et al. (2004, 2010).

We proceed in entirely different lines with the existing literature. In particular, we utilize some basic properties of the Sobolev

\footnotetext{
* Corresponding author. Tel.: +90 3122901598; fax: +90 3122665140.

E-mail addresses: edogan@bilkent.edu.tr (E. Dogan),

Cuong.Le-Van@univ-paris1.fr (C. Le Van), csaglam@bilkent.edu.tr (C. Saglam).
}

space $W_{l o c}^{1,1}$, and treat the problem by the standard tools of the calculus of variations. Our approach allows us to avoid the strict assumption that the value function be twice continuously differentiable. Yet, we are able to cover the three important aspects of the regime switching problems that have not been considered at the same time in the literature mentioned above: the infinite horizon for the objective functional to be maximized, the possibility of multiple regime switches and the explicit dependence of the constraint functions and the objective functional on these switching instants.

Except for the switching in the technology regime and the objective functional, our optimization framework is identical to the so-called reduced form optimal growth models which have been extensively used in economics due to their simple mathematical structure and generality (see McKenzie, 1986; Stokey and Lucas, 1989). Our crucial choice of the topological space is relevant for many optimal growth models, e.g. the Ramsey model, in which the feasible capital paths are proved to belong to this space and the feasible consumption paths belong to $L^{1}$ (see Askenazy and Le Van, 1999, page 42). The Sobolev space $W_{\text {loc }}^{1,1}$ also turns out to be a powerful tool to extract the usual transversality conditions as necessary optimality conditions for such infinite horizon optimal growth problems (see Le Van et al., 2007). Combining these with the standard tools of calculus of variations gets through the control problem of multiple regime switches without needing to decompose it in many auxiliary problems in a simple and unified 
manner. We prove that, in addition to the standard optimality conditions such as Euler-Lagrange, two specific sets of necessary conditions that characterize the optimal timing of regime switches emerge: continuity and the matching conditions. These are nothing but extensions of the Weierstrass-Erdmann corner conditions. Indeed, we show that Weierstrass-Erdmann corner conditions extend to the problems with switches.

In order to show how our approach allows us to derive properly and easily the necessary conditions for an infinite horizon multi-stage problem depending explicitly on the switching instant, we first analyze the optimal timing of technology adoption under embodiment and exogenously growing technology frontier. We show that the optimal timing of a technology upgrade depends crucially on how the growth advantage deriving from switching to a new economy with a higher degree of embodiment compares to the resulting obsolescence cost and the technology specific expertise loss. Later, we analyze an environmental control problem à la Boucekkine et al. (2010) that considers the trade-off between economic performance and environmental quality from the perspective of a government over a finite time horizon.

The paper is organized as follows. Section 2 presents the considered optimization problem, derives our necessary conditions of optimality for a two-stage problem, and compares them with the existing literature. Section 3 extends these results to the case of multiple regime switches. Section 4 provides applications to an optimal adoption problem under embodiment with exogenously growing technology frontier and an environmental control problem with the trade-off between economic performance and environmental quality. Finally, Section 5 concludes.

\section{Model}

We consider the optimal timing of switching between alternative and consecutive regimes in a continuous time reduced form model:

$\max$

$x(),. t_{1}$

subject to

$$
\begin{aligned}
& \int_{t_{0}}^{t_{1}} V^{1}\left(x(t), \dot{x}(t), t, t_{1}\right) \mathrm{e}^{-r t} \mathrm{~d} t \\
& +\int_{t_{1}}^{t_{f}} V^{2}\left(x(t), \dot{x}(t), t, t_{1}\right) \mathrm{e}^{-r t} \mathrm{~d} t
\end{aligned}
$$

$$
\begin{aligned}
& x\left(t_{0}\right)=x_{0}, \\
& (x(t), \dot{x}(t)) \in D_{t_{1}}(t) \subset \mathbb{R}^{2}, x(t) \geq 0, \text { a.e. on } \\
& {\left[t_{0}, t_{f}\right], t_{f} \leq \infty,}
\end{aligned}
$$

where $D_{t_{1}}(t)=\left\{(x, y) \mid \begin{array}{l}f^{1}\left(x, y, t, t_{1}\right) \geq 0, \text { for } t_{0} \leq t<t_{1} \\ f^{2}\left(x, y, t, t_{1}\right) \geq 0, \text { for } t_{f} \geq t>t_{1}\end{array}\right\}$, and $f^{i}$ are $\mathbb{R}^{m}$ valued, for $m \geq 1$. Throughout, we adapt the notation that the symbol $\geq$ denotes "all components are greater than or equal to...", and $>$ denotes "all components are strictly greater than...".

We recall some of the general definitions, notations and the results that will be useful in our analysis from Brezis (1983). We will say that a measurable function, $x:\left[t_{0}, t_{f}\right] \rightarrow \mathbb{R}$ is locally integrable if $|x|$ is integrable on any bounded interval and write $x \in L_{l o c}^{1}$. $L_{\text {loc }}^{\infty}$ will denote functions essentially bounded on finite intervals. By $C_{c}^{k}(a, b)$, we denote the set of $k$ times continuously differentiable functions, say $x$, in an open interval $(a, b)$ with $\operatorname{supp} x=\overline{\left\{t \in \mathbb{R}_{+}:|x(t)|>0\right\}} \subset(a, b)$. For any $x \in L_{l o c}^{1}, x^{\prime}$ is the weak derivative of $x$ if $\forall h \in C_{c}^{1}\left(t_{0}, t_{f}\right), \int_{t_{0}}^{t_{f}} x(t) \dot{h}(t) \mathrm{d} t=$ $-\int_{t_{0}}^{t_{f}} x^{\prime}(t) h(t) \mathrm{d} t$. For a function $x \in C_{c}^{1}\left(t_{0}, t_{f}\right)$, the weak derivative is identical with the ordinary derivative.

$W^{1,1} \equiv W^{1,1}\left(t_{0}, t_{f}\right) \equiv\left\{x \in L^{1} \quad: \quad x^{\prime}\right.$ exists and $x^{\prime} \in$ $\left.L^{1}\right\}$ with the norm defined by $\|x\|=\int_{t_{0}}^{t_{f}}|x| \mathrm{d} t+\int_{t_{0}}^{t_{f}}\left|x^{\prime}\right| \mathrm{d} t$, is the Sobolev space that we will be frequently referring to in our analysis. $W_{l o c}^{1,1}$ is similarly defined on $\left(t_{0}, t_{f}\right)$ to be $\{x \in$
$L_{l o c}^{1}: x^{\prime}$ exists and $\left.x^{\prime} \in L_{l o c}^{1}\right\}$. Two important properties of the Sobolev space will prove to be crucial in our analysis. As the elements of this space are equivalence classes, for any function $x \in$ $W^{1,1}$, there is a continuous representative $\tilde{x}$ which is equal to $x$ almost everywhere. We will be talking about this representative, whenever we refer to an element of this space. Secondly, weak derivative coincides with the usual derivative almost everywhere and $\tilde{x}(b)=\tilde{x}(a)+\int_{a}^{b} x^{\prime} \mathrm{d} t$. Thus, the elements of this space are absolutely continuous functions on finite intervals. In fact, on a finite open interval, the set of absolutely continuous functions and the Sobolev space $W^{1,1}$ are the same.

Definition 1. A pair $\left(\underset{\sim}{x}(),.{\underset{\sim}{1}}_{1}\right)$ is admissible if $\underset{\sim}{x}(t) \in W_{l o c}^{1,1}, \underset{\sim}{\dot{x}}(t) \in$ $L_{l o c}^{\infty}$, satisfy the constraints

$\underset{\sim}{x}\left(t_{0}\right)=x_{0}$,

$\underset{\sim}{(x}(t), \underset{\sim}{\dot{x}}(t)) \in D_{t_{1}}(t) \subset \mathbb{R}^{2}, \quad \underset{\sim}{x}(t) \geq 0$,

a.e. on $\left[t_{0}, t_{f}\right], t_{f} \leq+\infty$, and

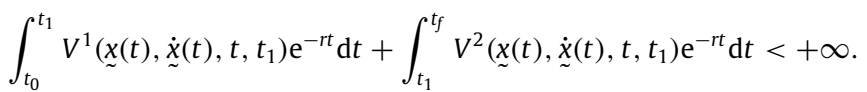

A pair $\left(x(),. t_{1}\right)$ is an optimal solution if it is admissible and if the value of the objective function corresponding to any admissible pair is not greater than that of $\left(x(),. t_{1}\right)$.

From now on, $x$ will always refer to the optimal values unless otherwise stated. We have the following set of assumptions.

Assumption 1. $V^{i}: \mathbb{R}^{4} \rightarrow \mathbb{R}$ is $C^{1}$ and $f^{i}: \mathbb{R}^{4} \rightarrow \mathbb{R}^{m}$ is continuous for $i=1,2$.

Assumption 2 (Interiority). $x(t)>0, f^{i}\left(x, \dot{x}, t, t_{1}\right)>0$ uniformly in the sense of the space $L^{\infty}$ on any bounded interval for $i=1,2$ (i.e., on any bounded interval there exists an $\varepsilon>0$ such that $x(t)>\varepsilon, f^{i}\left(x, \dot{x}, t, t_{1}\right)>\varepsilon$, on their respective domains, almost everywhere on the interval).

The following proposition gives the Euler-Lagrange equation for the problem that incorporates a change in the objective functional at an instant in a very elementary way within our functional framework. To ease the notation, the third and the fourth argument of $V^{i}(i=1,2)$ will be suppressed whenever we do not need them.

Proposition 1 (Euler-Lagrange). Under Assumptions 1 and 2, the optimal $x(t)$ satisfies

$\left(V_{\dot{x}}(x, \dot{x}) \mathrm{e}^{-r t}\right)^{\prime}=V_{x}(x, \dot{x}) \mathrm{e}^{-r t}$,

almost everywhere on any bounded interval $(a, b)$, where $V$ should be read as $V^{1}$ whenever $t<t_{1}$ and $V^{2}$ whenever $t>t_{1}$.

Proof. The proof follows from Dana and Le Van (2003), but it is based on the use of weak derivatives to handle the switching between alternative regimes.

Consider any bounded interval $(a, b)$ on $\left(t_{0}, t_{f}\right)$. Take any $h \in$ $C_{c}^{1}(a, b)$, and assume that it is extended to zero outside of $(a, b)$. For $|\lambda|$ small $x+\lambda h>0$, clearly. Moreover, for $|\lambda|$ small, for an appropriate $\epsilon,(x+\lambda h, \dot{x}+\lambda \dot{h})$ is in an open ball of radius $\epsilon$ centered at $(x, \dot{x})$, for each $t \in(a, b)$ so that $f^{i}\left(x+\lambda h, \dot{x}+\lambda \dot{h}, t, t_{1}\right)>0$, for $i=1,2$.

Define $\varphi(\lambda)=\int_{a}^{b} V(x+\lambda h, \dot{x}+\lambda \dot{h}) \mathrm{e}^{-r t} \mathrm{~d} t=\varphi_{1}(\lambda)+\varphi_{2}(\lambda)$, and write $\varphi_{1}(\lambda)=\int_{a}^{t_{1}} V^{1}(x+\lambda h, \dot{x}+\lambda \dot{h}) \mathrm{e}^{-r t} \mathrm{~d} t, \varphi_{2}(\lambda)=\int_{t_{1}}^{b} V^{2}(x+$ $\lambda h, \dot{x}+\lambda \dot{h}) \mathrm{e}^{-r t} \mathrm{~d} t$. For any sequence of real numbers $\lambda_{n} \rightarrow 0$, fixing any $t$,

$$
\begin{aligned}
\frac{V\left(x+\lambda_{n} h, \dot{x}+\lambda_{n} \dot{h}\right)-V(x, \dot{x})}{\lambda_{n}}= & V_{x}\left(x+\bar{\lambda}_{n} h, \dot{x}+\bar{\lambda}_{n} \dot{h}\right) h \\
& +V_{\dot{x}}\left(x+\bar{\lambda}_{n} h, \dot{x}+\bar{\lambda}_{n} \dot{h}\right) \dot{h},
\end{aligned}
$$

for some $0<\left|\bar{\lambda}_{n}\right|<\left|\lambda_{n}\right|$, by Mean Value Theorem. 
Now, $V_{x}$ and $V_{\dot{x}}$ are continuous and they are restricted to a bounded rectangle in $\mathbb{R}^{2}$, due to the continuity of $x$ and the boundedness of $\dot{x}$. So, $V_{x}\left(x+\bar{\lambda}_{n} h, \dot{x}+\bar{\lambda}_{n} \dot{h}\right)$ and $V_{\dot{x}}\left(x+\bar{\lambda}_{n} h, \dot{x}+\bar{\lambda}_{n} \dot{h}\right) \dot{h}$ are bounded in $L^{\infty}(a, b)$ when $n$ is large enough.

Thus, there exists $K \in \mathbb{R}$, such that $\left|\frac{V\left(x+\lambda_{n} h, \dot{x}+\lambda_{n} \dot{h}\right)-V(x, \dot{x})}{\lambda_{n}}\right| \leq$ $K$, a.e. on $(a, b)$. Then, we may apply Dominated Convergence Theorem to the sequence

$\frac{\varphi_{1}\left(\lambda_{n}\right)-\varphi_{1}(0)}{\lambda_{n}}=\int_{a}^{t_{1}} \frac{V^{1}\left(x+\lambda_{n} h, \dot{x}+\lambda_{n} \dot{h}\right)-V^{1}(x, \dot{x})}{\lambda_{n}} \mathrm{e}^{-r t} \mathrm{~d} t$,

concluding that $\varphi_{1}(\lambda)$ is differentiable at 0 with the derivative,

$$
\begin{aligned}
& \lim _{n \rightarrow \infty} \int_{a}^{t_{1}} \frac{V^{1}\left(x+\lambda_{n} h, \dot{x}+\lambda_{n} \dot{h}\right)-V^{1}(x, \dot{x})}{\lambda_{n}} \mathrm{e}^{-r t} \mathrm{~d} t \\
& =\int_{a}^{t_{1}}\left(V_{x}^{1}(x, \dot{x}) h \mathrm{e}^{-r t}+V_{\dot{x}}^{1}(x, \dot{x}) \dot{h} \mathrm{e}^{-r t}\right) \mathrm{d} t .
\end{aligned}
$$

By repeating the same steps on $\left(t_{1}, b\right)$ one may also find that $\varphi_{2}^{\prime}(0)=\int_{t_{1}}^{b}\left(V_{x}^{2}(x, \dot{x}) h \mathrm{e}^{-r t}+V_{\dot{x}}^{2}(x, \dot{x}) \dot{h} \mathrm{e}^{-r t}\right) \mathrm{d} t$.

Hence, we easily obtain that $\varphi^{\prime}(0)=\int_{a}^{b}\left(V_{x}(x, \dot{x}) h \mathrm{e}^{-r t}+V_{\dot{x}}(x, \dot{x}) \dot{h} \mathrm{e}^{-r t}\right) \mathrm{d} t$.

Now, $\int_{a}^{b} V(x+\lambda h, \dot{x}+\lambda \dot{h}) \mathrm{e}^{-r t} \mathrm{~d} t-\int_{a}^{b} V(x, \dot{x}) \mathrm{e}^{-r t} \mathrm{~d} t=\varphi(\lambda)-$ $\varphi(0)$, so that $\varphi($.) is maximized at 0 . Since $\varphi($.$) is differentiable at$ zero,

$\varphi^{\prime}(0)=\int_{a}^{b}\left(V_{x}(x, \dot{x}) \mathrm{e}^{-r t} h+V_{\dot{x}}(x, \dot{x}) \mathrm{e}^{-r t} \dot{h}\right) \mathrm{d} t=0$.

As $h \in C_{c}^{1}(a, b)$ was arbitrary, $\left(V_{\dot{x}}(x, \dot{x}) \mathrm{e}^{-r t}\right)^{\prime}=V_{x}(x, \dot{x}) \mathrm{e}^{-r t}$, i.e. $V_{x}(x, \dot{x}) \mathrm{e}^{-r t}$ is the weak derivative of $V_{\dot{x}}(x, \dot{x}) \mathrm{e}^{-r t}$ on $(a, b)$.

By means of the Euler-Lagrange equation, we are able to derive an important result for the problems with switches, known as the first Weierstrass-Erdmann condition.

Corollary 1 (Continuity Condition). Let Assumptions 1 and 2 be satisfied. Then $V_{\dot{x}}(x, \dot{x}) \mathrm{e}^{-r t}$ is continuous everywhere, and in particular, at the switching instant.

Proof. The Euler-Lagrange equation implies $V_{\dot{x}}(x, \dot{x}) \mathrm{e}^{-r t} \in W_{l o c}^{1,1}$ so that $V_{\dot{x}}(x, \dot{x}) \mathrm{e}^{-r t}$ is absolutely continuous on any bounded interval and hence continuous everywhere.

The following results and the set of assumptions that impose more regularity on $x(t)$, will be crucial in establishing the optimality conditions with respect to the switching instant.

Corollary 2. The optimal $x(t)$ is locally Lipschitz, i.e., Lipschitz on any bounded interval.

Proof. Since $x(t)$ is admissible, $|\dot{x}(t)|$ is bounded locally. Hence, for any bounded $(a, b) \subset\left(t_{0}, t_{f}\right)$, there is some $K$ such that for all $t \in(a, b),|\dot{x}(t)| \leq K$ and thus $|x(b)-x(a)|=\left|\int_{a}^{b} \dot{x} \mathrm{~d} t\right| \leq$ $K|b-a|$.

In what follows, some global properties of the functions $V^{i}$ ( $i=1,2$ ) will be needed. Because of this, we continue with the following modification of Assumption 1. We write $V_{2}^{i}$ for the derivative of $V^{i}$ with respect to the second variable, and $V_{22}^{i}$ for the derivative of $V_{2}^{i}$ with respect to the second variable.

Assumption 3. $V_{2}^{i}$ is $C^{1}$ and $V_{22}^{i}$ is invertible (i.e., either $V_{22}^{i}<0$ or $V_{22}^{i}>0$ ) on $\mathbb{R} \times \mathbb{R} \times\left[t, t^{\prime}\right]$ for $t, t^{\prime}$ finite in $\left[t_{0}, t_{f}\right]$, and $i=1,2$.

Proposition 2. If the optimal $x$ is Lipschitz on bounded open intervals, then $x$ is $C^{2}$ except possibly at $t_{1}$.

Proof. See Buttazzo et al. (1998), Proposition 4.4, page 135.
Note that Assumption 3 assumes a global invertibility condition, which may be violated in applications. If, however, the solution of the Euler-Lagrange equation happens to be $C^{1}$ then one may utilize a local invertibility criterion as the following variant of Proposition 2 demonstrates.

Proposition 3. For any bounded interval $I$, if $V_{2}^{i}$ is $C^{1}$ on some neighborhood of the path $(x, \dot{x}, t), V_{22}^{i}$ is invertible along the path $(x, \dot{x}, t)$, for $t \in I, i=1,2$, and $x$ is $C^{1}$ (except possibly at $t_{1}$ ), then $x$ is $C^{2}$ (except possibly at $t_{1}$ ).

Proof. See Buttazzo et al. (1998), Proposition 4.2, page 135.

So whenever global invertibility and smoothness conditions of Assumption 3 are violated one may replace Assumption 3 with the assumptions of Proposition 3. In this case, one may also restrict the domain of Assumption 1 to a small enough neighborhood around the optimal path, if necessary. This simply follows from the fact that the proof of the Euler-Lagrange equation utilizes the assumption only in such a neighborhood. In fact, it is this version that we utilize in the technology adoption and the environmental control problems presented in Section 4.

Assumption 4. There exists an integrable function $g(t)$ on $\left[t_{0}, t_{f}\right]$ and some interval $I \subset\left[t_{0}, t_{f}\right]$, such that $t_{1}$ is in the interior of $I$, and $\forall s \in I, \forall t,\left|V_{s}^{i}(x, \dot{x}, t, s)\right| \mathrm{e}^{-r t} \leq g(t)$, for $i \in\{1,2\}$ (in the case of $t_{1}=\infty$, the interval $I$ is of the form, $[N,+\infty)$ for some $\left.N<+\infty\right)$.

Note that if the planning horizon is finite, i.e., $t_{f}<\infty$, Assumption 5 is automatically satisfied. The next proposition, which is a variant of the second Weierstrass-Erdmann corner conditions, will be proved under Assumptions 1-4, by the socalled "variation of the independent variable" technique. In the next proposition, recall also that Assumption 3 can be replaced with the assumptions of Proposition 3, and Assumption 1 can be replaced to be satisfied in a neighborhood of the optimal path, whenever convenient.

Proposition 4 (Matching Condition). Under Assumptions 1-4, optimal pair $\left(x, t_{1}\right)$ satisfies

$$
\begin{gathered}
{\left[\dot{x} V_{\dot{x}}^{1}-V^{1}\right]_{t_{1}} \mathrm{e}^{-r t_{1}}-\left[\dot{x} V_{\dot{x}}^{2}-V^{2}\right]_{t_{1}} \mathrm{e}^{-r t_{1}}} \\
=\int_{t_{0}}^{t_{1}} V_{t_{1}}^{1} \mathrm{e}^{-r t} \mathrm{~d} t+\int_{t_{1}}^{t_{f}} V_{t_{1}}^{2} \mathrm{e}^{-r t} \mathrm{~d} t
\end{gathered}
$$

whenever $t_{0}<t_{1}<t_{f}$.

Proof. Take any $h \in C_{c}^{1}\left(t_{0}, t_{f}\right)$, and define a function $\tau(t, \epsilon)=$ $t-\epsilon h(t)$ on $\left[t_{0}, t_{f}\right]$ ( $h$ is extended to zero outside $\left(t_{0}, t_{f}\right)$ ). Note that $\tau\left(t_{0}, \varepsilon\right)=t_{0}$ and $\tau\left(t_{f}, \varepsilon\right)=t_{f}$. For $|\epsilon|$ small enough, $\tau_{t}(t, \epsilon)=$ $1-\epsilon h^{\prime}(t)>0$ (we continue to use subscripts for derivatives). Thus, for all such small $|\epsilon|$, the mapping $\tau(., \epsilon)$ is a $C^{1}$ diffeomorphism of $\left[t_{0}, t_{f}\right]$. Write $\zeta(s, \epsilon)$, for the inverse of this mapping, and denote $\tau\left(t_{1}, \epsilon\right)=s_{1}$.

Since the transformation $t \mapsto t-\epsilon h(t)$, is monotonic, for $|\epsilon|$ small enough, the path $x(\zeta(s, \epsilon))$ as a function of $s=$ $\tau(t, \epsilon)$, satisfies the constraints of the problem, thanks to the differentiability properties of the functions and continuity (expect possibly for the switching instant) of the solutions involved. Let $W^{i}\left(x, \dot{x}, t, t_{1}\right)=V^{i}\left(x, \dot{x}, t, t_{1}\right) \mathrm{e}^{-r t}, i=1,2$. So,

$$
\begin{aligned}
\varphi(\epsilon)= & \int_{t_{0}}^{s_{1}} W^{1}\left(x(\zeta(s, \epsilon)), \frac{\mathrm{d} x(\zeta(s, \epsilon))}{\mathrm{d} s}, s, s_{1}\right) \mathrm{d} s \\
& +\int_{s_{1}}^{t_{f}} W^{2}\left(x(\zeta(s, \epsilon)), \frac{\mathrm{d} x(\zeta(s, \epsilon))}{\mathrm{d} s}, s, s_{1}\right) \mathrm{d} s
\end{aligned}
$$

is maximized at 0 (Note that $\tau(t, 0)=t$ ). 
Since $\frac{\mathrm{d} x(\zeta(s, \epsilon))}{\mathrm{d} s}=\dot{x}(\zeta(s, \epsilon)) \zeta_{s}(s, \epsilon)$, we write:

$$
\begin{aligned}
\varphi(\epsilon)= & \int_{t_{0}}^{s_{1}} W^{1}\left(x(\zeta(s, \epsilon)), \dot{x}(\zeta(s, \epsilon)) \zeta_{s}(s, \epsilon), s, s_{1}\right) \mathrm{d} s \\
& +\int_{s_{1}}^{t_{f}} W^{2}\left(x(\zeta(s, \epsilon)), \dot{x}(\zeta(s, \epsilon)) \zeta_{s}(s, \epsilon), s, s_{1}\right) \mathrm{d} s .
\end{aligned}
$$

As $\varphi(\epsilon)$ is finite and $\tau$ is a $C^{1}$ diffeomorphism, the change of variables (see Lang, 1993, p. 505, Theorem 2.6) allows us to transform this equation into the following form:

$$
\begin{gathered}
\varphi(\epsilon)=\int_{t_{0}}^{t_{1}} W^{1}\left(x(t), \dot{x}(t) \frac{1}{\tau_{t}(t, \epsilon)}, \tau(t, \epsilon), \tau\left(t_{1}, \epsilon\right)\right) \tau_{t}(t, \epsilon) \mathrm{d} t \\
+\int_{t_{1}}^{t_{f}} W^{2}\left(x(t), \dot{x}(t) \frac{1}{\tau_{t}(t, \epsilon)}, \tau(t, \epsilon), \tau\left(t_{1}, \epsilon\right)\right) \tau_{t}(t, \epsilon) \mathrm{d} t
\end{gathered}
$$

where we use $\tau_{t}(\zeta(s, \epsilon), \epsilon) \zeta_{s}(s, \epsilon)=1$.

Now, in a neighborhood of zero, by Assumptions 1 and 4, the partial derivatives with respect to $\epsilon$ of the integrands above,

$\left(1-\epsilon h^{\prime}\right)\left[-W_{t}^{i} h+\dot{x} W_{\dot{x}}^{i} \frac{h^{\prime}}{\left(1-\epsilon h^{\prime}\right)^{2}}-W_{t_{1}}^{i} h\left(t_{1}\right)\right]-W^{i} h^{\prime}$,

will be dominated by an integrable function. This is obvious for the terms multiplied by $h$ or $h^{\prime}$. For the term, $\left(1-\epsilon h^{\prime}\right) W_{t_{1}}^{i} h\left(t_{1}\right)$, this is due to the fact that for $\varepsilon$ small, $\tau\left(t_{1}, \varepsilon\right)$ will be in the interval $I$ from Assumption 4, so that some $g(t)$ dominates the term $\left|W_{t_{1}}^{i}\right|$, while $\left|\left(1-\epsilon h^{\prime}\right) h\left(t_{1}\right)\right|$ is already bounded on $\left[t_{0}, t_{f}\right]$. It then follows by the dominated convergence theorem that $\varphi(\epsilon)$ is differentiable at zero. This derivative is equal to zero, and is given by the following expression (we suppress the arguments of the functions):

$$
\begin{aligned}
\varphi^{\prime}(0)= & \int_{t_{0}}^{t_{1}}\left[-W_{t}^{1} h+\dot{x} W_{\dot{x}}^{1} h^{\prime}-W_{t_{1}}^{1} h\left(t_{1}\right)-W^{1} h^{\prime}\right] \mathrm{d} t \\
& +\int_{t_{1}}^{t_{f}}\left[-W_{t}^{2} h+\dot{x} W_{\dot{x}}^{2} h^{\prime}-W_{t_{1}}^{2} h\left(t_{1}\right)-W^{2} h^{\prime}\right] \mathrm{d} t .
\end{aligned}
$$

By integration by parts, we obtain

$$
\begin{aligned}
\int_{t_{0}}^{t_{1}}\left[\dot{x} W_{\dot{x}}^{1}-W^{1}\right] h^{\prime} \mathrm{d} t= & {\left[\dot{x} W_{\dot{x}}^{1}-W^{1}\right]_{t_{1}} h\left(t_{1}\right) } \\
& -\int_{t_{0}}^{t_{1}} \frac{\mathrm{d}\left[\dot{x} W_{\dot{x}}^{1}-W^{1}\right]}{\mathrm{d} t} h \mathrm{~d} t, \\
\int_{t_{1}}^{t_{f}}\left[\dot{x} W_{\dot{x}}^{2}-W^{2}\right] h^{\prime} \mathrm{d} t= & -\left[\dot{x} W_{\dot{x}}^{2}-W^{2}\right]_{t_{1}} h\left(t_{1}\right) \\
& -\int_{t_{1}}^{t_{f}} \frac{\mathrm{d}\left[\dot{x} W_{\dot{x}}^{2}-W^{2}\right]}{\mathrm{d} t} h \mathrm{~d} t .
\end{aligned}
$$

Plugging these in $\varphi^{\prime}(0)$, we obtain

$$
\begin{aligned}
& h\left(t_{1}\right)\left(\left[\dot{x} W_{\dot{x}}^{1}-W^{1}\right]_{t_{1}}-\left[\dot{x} W_{\dot{x}}^{2}-W^{2}\right]_{t_{1}}\right) \\
& \quad+\int_{t_{0}}^{t_{1}}\left(-W_{t}^{1}-\frac{\mathrm{d}\left[\dot{x} W_{\dot{x}}^{1}-W^{1}\right]}{\mathrm{d} t}\right) h \mathrm{~d} t \\
& \quad+\int_{t_{1}}^{t_{f}}\left(-W_{t}^{2}-\frac{\mathrm{d}\left[\dot{x} W_{\dot{x}}^{2}-W^{2}\right]}{\mathrm{d} t}\right) h \mathrm{~d} t \\
& =h\left(t_{1}\right)\left(\int_{t_{0}}^{t_{1}} W_{t_{1}}^{1} \mathrm{~d} t+\int_{t_{1}}^{t_{f}} W_{t_{1}}^{2} \mathrm{~d} t\right) .
\end{aligned}
$$

For $h\left(t_{1}\right) \neq 0$,

$$
\begin{gathered}
{\left[\dot{x} W_{\dot{x}}^{1}-W^{1}\right]_{t_{1}}-\left[\dot{x} W_{\dot{x}}^{2}-W^{2}\right]_{t_{1}}} \\
=\int_{t_{0}}^{t_{1}} W_{t_{1}}^{1} \mathrm{~d} t+\int_{t_{1}}^{t_{f}} W_{t_{1}}^{2} \mathrm{~d} t
\end{gathered}
$$

$$
\begin{aligned}
& +\frac{1}{h\left(t_{1}\right)}\left[\int_{t_{0}}^{t_{1}}\left(W_{t}^{1}+\frac{\mathrm{d}\left[\dot{x} W_{\dot{x}}^{1}-W^{1}\right]}{\mathrm{d} t}\right) h \mathrm{~d} t\right. \\
& \left.+\int_{t_{1}}^{t_{f}}\left(W_{t}^{2}+\frac{\mathrm{d}\left[\dot{x} W_{\dot{x}}^{2}-W^{2}\right]}{\mathrm{d} t}\right) h \mathrm{~d} t\right] .
\end{aligned}
$$

We will now prove that $W_{t}^{1}+\frac{\mathrm{d}\left[\dot{x} W_{\dot{x}}^{1}-W^{1}\right]}{\mathrm{d} t}=0$. Indeed, since $\frac{\mathrm{d}\left(W_{\dot{x}}^{1}\right)}{\mathrm{d} t}=W_{x}^{1}$ by the Euler equation, one has

$$
\begin{aligned}
\frac{\mathrm{d}\left[\dot{x} W_{\dot{x}}^{1}-W^{1}\right]}{\mathrm{d} t} & =\ddot{x} W_{\dot{x}}^{1}+\dot{x} W_{x}^{1}-W_{x}^{1} \dot{x}-W_{\dot{x}}^{1} \ddot{x}-W_{t}^{1} \\
& =-W_{t}^{1} .
\end{aligned}
$$

The result follows. Similarly, one gets

$W_{t}^{2}+\frac{\mathrm{d}\left[\dot{x} W_{\dot{x}}^{2}-W^{2}\right]}{\mathrm{d} t}=0$.

Therefore, replacing $W^{i}$ by $V^{i} \mathrm{e}^{-r t}$ in (7) gives (3).

In order to consider the corner solution cases in which the optimal switching time is at one of the terminal times, we need an additional assumption ensuring that some initial or final segment of an optimal path $x$, is also admissible under the other regime. Note that, whenever $t_{1}$ is an interior point of $\left[t_{0}, t_{f}\right]$, such a uniformity requirement is not necessary at all, as the inner variation of the optimal path around an interior switching point respects the admissibility condition anyway.

Assumption 5. Let $\left(x, t_{1}\right)$ be an optimal pair. If $t_{1}=t_{0}$, there exists a non-degenerate interval $t_{0} \ni I \subset\left[t_{0}, t_{f}\right]$ and $\epsilon>0$, such that, $\forall s \in I$, and $t<s, f^{1}(x(t), \dot{x}(t), t, s)>\epsilon$. If $t_{1}=t_{f}$, there exists a non-degenerate interval $t_{f} \ni I \subset\left[t_{0}, t_{f}\right]$ and $\epsilon>0$, such that, $\forall s \in I, \exists \bar{t}$ such that, if $t>s, f^{2}(x(t), \dot{x}(t), t, s) \geq 0$ and if $\bar{t}>t>s, f^{2}(x(t), \dot{x}(t), t, s)>\epsilon$ (note that we need $f^{1}(x(t), \dot{x}(t), t, s)>\epsilon$ on $\left(t_{0}, s\right)$ and $f^{2}(x(t), \dot{x}(t), t, s)>\epsilon$ on $(s, \bar{t})$ in order to allow room for inner variation on finite intervals around the switching point).

Proposition 5. Under Assumptions 1-5, whenever the optimal switching time is at one of the terminal times, the matching condition should be modified as

$$
\begin{gathered}
{\left[\dot{x} V_{\dot{x}}^{1}-V^{1}\right]_{t=t_{0}} \mathrm{e}^{-r t_{0}}-\left[\dot{x} V_{\dot{x}}^{2}-V^{2}\right]_{t=t_{0}} \mathrm{e}^{-r t_{0}}} \\
\geq \int_{t_{0}}^{t_{f}} V_{t_{1}}^{2} \mathrm{e}^{-r t} \mathrm{~d} t, \text { for } t_{1}=t_{0}, \text { and } \\
{\left[\dot{x} V_{\dot{x}}^{1}-V^{1}\right]_{t=t_{f}} \mathrm{e}^{-r t_{f}}-\left[\dot{x} V_{\dot{x}}^{2}-V^{2}\right]_{t=t_{f}} \mathrm{e}^{-r t_{f}}} \\
\leq \int_{t_{0}}^{t_{f}} V_{t_{1}}^{1} \mathrm{e}^{-r t} \mathrm{~d} t, \quad \text { for } t_{1}=t_{f},
\end{gathered}
$$

where in the case of $t_{f}=\infty$, the last inequality holds in the limit.

Proof. The proof follows from the calculation of the limit of a directional derivative of the function $\varphi(\epsilon)$, which is defined in the proof of Proposition 4, where the limit is taken with respect to a sequence of functions $h_{n}$ replacing $h$ in $\varphi(\epsilon)$. But this calculation is rather tedious and we omit it.

Remark 1. In order to compare our results with those of the twostage optimal control approach, define the Hamiltonian of the preswitch and post-switch phases of the problem as

$H^{i}\left(x, p, t, t_{1}\right)=-V^{i}\left(x, \dot{x}, t, t_{1}\right) \mathrm{e}^{-r t}+p^{i} \dot{x}, \quad i=1,2$.

Following from Dana and Le Van (2003), under the conditions that $V^{i}$ is $C^{2}, V_{22}^{i}$ is invertible, say $V_{22}^{i}<0$, for $i=1,2$, a solution of the Euler-Lagrange equation is a solution of the corresponding 
Hamiltonian system, i.e., the equation system: $\frac{\partial H^{i}}{\partial p}=\dot{x}, \frac{\partial H^{i}}{\partial x}=-\dot{p}^{i}$, and vice versa. Moreover, note that $V_{\dot{x}}\left(x, \dot{x}, t, t_{1}\right) \mathrm{e}^{-r t}=p(t)$, at any $t$, and $\left.H^{2}\right|_{t_{1}}=\left[\dot{x} V_{\dot{x}}^{2}-V^{2}\right]_{t_{1}} \mathrm{e}^{-r t_{1}},\left.H^{1}\right|_{t_{1}}=\left[\dot{x} V_{\dot{x}}^{1}-V^{1}\right]_{t_{1}} \mathrm{e}^{-r t_{1}}$ (see Buttazzo et al., 1998, Proposition 1.34, p. 38). These establish the continuity of the co-state variable at the switching instant and the following matching condition for an interior switch stated in Tomiyama and Rossana (1989):

$\left[\left.H^{2}\right|_{t_{1}}\right]-\left[\left.H^{1}\right|_{t_{1}}\right]-\int_{t_{0}}^{t_{1}} \frac{\partial H^{1}}{\partial t_{1}} \mathrm{~d} t-\int_{t_{1}}^{t_{f}} \frac{\partial H^{2}}{\partial t_{1}} \mathrm{~d} t=0$.

Remark 2. When the switching instant does not appear explicitly in the integrands or the constraints of the problem, it is clear that the matching condition reduces to $\left[\left.H^{2}\right|_{t_{1}}\right]=\left[\left.H^{1}\right|_{t_{1}}\right]$, as stated in Makris (2001) and Tomiyama (1985).

\section{Multiple regime switches}

These results can easily be generalized to consider the problems with multiple regime switches. In this respect, consider the following problem with $f-1$ switches.

$\max _{x(t), t_{1}} \quad \sum_{k=1}^{f} \int_{t_{k-1}}^{t_{k}} V^{k}\left(x(t), \dot{x}(t), t, t_{1}, t_{2}, \ldots, t_{f-1}\right) \mathrm{e}^{-r t} \mathrm{~d} t$

subject to

$$
\begin{aligned}
& (x(t), \dot{x}(t)) \in D_{t_{1}, t_{2}, \ldots, t_{f-1}}(t) \subset \mathbb{R}^{2}, \\
& x\left(t_{0}\right)=x_{0}, \quad x(t) \geq 0, \\
& \text { a.e. on }\left[t_{0}, t_{f}\right], t_{f} \leq \infty,
\end{aligned}
$$

where $D_{t_{1}, t_{2}, \ldots, t_{f-1}}(t)=\left\{(x, y) \mid f^{k}\left(x, y, t, t_{1}, t_{2}, \ldots, t_{f-1}\right) \geq\right.$ 0 , for $\left.t_{k-1} \leq t<t_{k}, \forall k=1,2, \ldots, f\right\}$.

The novel feature of this problem with multiple regime switches is that the endogenous switching instants appear explicitly as an argument of the law of motion of the state and the objective criteria. It is important to note that early contributions by Tomiyama (1985), Tomiyama and Rossana (1989) and Makris (2001) cannot be used to handle this optimization problem.

It is clear that the assumptions for the single switch, the Euler-Lagrange equation, and hence the continuity condition extend immediately for such problems. In order to characterize the optimal timing of the multiple switching instants, one has to deal with the extension of the matching condition. Following the same steps in the proof of the single switch matching condition, one can rewrite (7) as

$$
\begin{aligned}
\varphi^{\prime}(0)= & \sum_{k=1}^{f}\left\{h\left(t_{k}\right)\left[\dot{x} W_{\dot{x}}^{k}-W^{k}\right]_{t_{k}}-h\left(t_{k-1}\right)\left[\dot{x} W_{\dot{x}}^{k}-W^{k}\right]_{t_{k-1}}\right. \\
& \left.-\int_{t_{k-1}}^{t_{k}}\left(\sum_{i=1}^{f-1}\left(h\left(t_{i}\right) W_{t_{i}}^{k}\right)+\phi^{k} h\right) \mathrm{d} t\right\},
\end{aligned}
$$

where $\phi^{i}(t) \equiv-V_{t}^{i}-\frac{\mathrm{d}\left[\dot{x} V_{\dot{x}}^{i}-V^{i}\right]}{\mathrm{d} t}$, for $i \in\{1,2, \ldots, f\}$.

For $t_{0}<t_{1}<t_{2}<\cdots<t_{f-1}<t_{f}$, we have $\varphi^{\prime}(0)=0$. Now, if $h$ is such that $h\left(t_{i}\right) \neq 0$ and $h\left(t_{j}\right)=0, \forall j \neq i$ (note that $h\left(t_{f}\right)=h\left(t_{0}\right)=0$, as $h$ will have compact support on $\left.\left(t_{0}, t_{f}\right)\right)$, then we obtain

$$
\begin{aligned}
& {\left[\dot{x} V_{\dot{x}}^{i}-V^{i}\right]_{t_{i}} \mathrm{e}^{-r t_{i}}-\left[\dot{x} V_{\dot{x}}^{i+1}-V^{i+1}\right]_{t_{i}} \mathrm{e}^{-r t_{i}}} \\
& \quad=\sum_{j=1}^{f}\left(\int_{t_{j-1}}^{t_{j}} V_{t_{i}}^{j} \mathrm{e}^{-r t} \mathrm{~d} t\right) .
\end{aligned}
$$

Similarly, the necessary conditions for $t_{1}, t_{2}, \ldots, t_{f-1}$ to be interior optimal switching instants can then be written as

$$
\begin{aligned}
& {\left[\dot{x} V_{\dot{x}}^{i}-V^{i}\right]_{t_{i}} \mathrm{e}^{-r t_{i}}-\left[\dot{x} V_{\dot{x}}^{i+1}-V^{i+1}\right]_{t_{i}} \mathrm{e}^{-r t_{i}}} \\
& \quad=\sum_{j=1}^{f}\left(\int_{t_{j-1}}^{t_{j}} V_{t_{i}}^{j} \mathrm{e}^{-r t} \mathrm{~d} t\right), \quad \forall i=1,2, \ldots, f-1 .
\end{aligned}
$$

In general, in such a system with $f-1$ switches, or equivalently in a system with $f$ possible regimes, one has to consider also $\frac{(3 f-4)(f-1)}{2}$ possible corner solution cases. ${ }^{1}$ As an example, let us work on a system that involves 2 regime switches and the following out of the four possible configurations: $t_{0}=t_{1}=$ $t_{2}<t_{f}$. In this case the system immediately jumps to the third stage. Considering the appropriate limits, we have the following as necessary conditions:

$$
\begin{aligned}
& {\left[\dot{x} V_{\dot{x}}^{1}-V^{1}\right]_{t=t_{0}} \mathrm{e}^{-r t_{0}}-\left[\dot{x} V_{\dot{x}}^{3}-V^{3}\right]_{t=t_{0}} \mathrm{e}^{-r t_{0}} \geq \int_{t_{0}}^{t_{f}} V_{t_{1}}^{3} \mathrm{e}^{-r t} \mathrm{~d} t} \\
& {\left[\dot{x} V_{\dot{x}}^{2}-V^{2}\right]_{t=t_{0}} \mathrm{e}^{-r t_{0}}-\left[\dot{x} V_{\dot{x}}^{3}-V^{3}\right]_{t=t_{0}} \mathrm{e}^{-r t_{0}} \geq \int_{t_{0}}^{t_{f}} V_{t_{2}}^{3} \mathrm{e}^{-r t} \mathrm{~d} t .}
\end{aligned}
$$

In this manner, the necessary conditions for all corner solutions can be written. But it is clear that implementing these in practice is really hard, as the number of necessary conditions grow very fast.

\section{Applications}

In this section, we consider two applications of our results. First, we shall solve a technology adoption problem with expanding technology frontier in order to show how our approach allows us to derive properly and easily the necessary conditions for an infinite horizon multi-stage problem depending explicitly on the switching instant. As advancement of technology may be regarded as a continuous process while the adoption of it is a discrete process, our analysis will be legitimate in its approach to the adoption problem. Yet, the analysis below should be treated as a complement to the studies of Boucekkine et al. (2004, 2010), as the adoption process is rather complicated with determinants like learning, network externalities, and strategic interactions, effects of which are studied by these authors. Second, we consider an environmental control problem. In this problem we illustrate how easy it is to obtain necessary and sufficient conditions for an interior switching time with the present approach.

\subsection{Optimal timing of technology adoption}

We consider the following technology adoption problem:

$\max _{k(t), t_{1}} \int_{0}^{\infty} \ln (c(t)) \mathrm{e}^{-\rho t} \mathrm{~d} t$

subject to

$\dot{k}(t)= \begin{cases}q(0)\left(a_{1} k(t)-c(t)\right), & \text { for } t<t_{1}, \\ q\left(t_{1}\right)\left(a_{2} k(t)-c(t)\right), & \text { for } t \geq t_{1},\end{cases}$

$k(0)=k_{0}>0, \quad c(t) \geq 0, \quad \dot{k}(t) \geq 0$,

where $c$ denotes the flow of consumption and $\rho$ is the time discounting parameter. The problem can easily be transformed into

\footnotetext{
1 This follows from the following argument: there are $\frac{f(f-1)}{2}$ corner cases corresponding to immediate jump to a higher regime at $t_{0}$; there are $\frac{(f-1)(f-2)}{2}$ corner cases corresponding to not switching to a higher regime (i.e. cases in which first regime forever, or second regime forever, or ...); there are $\frac{(f-1)(f-2)}{2}$ corner cases corresponding to nonswitching to an intermediate regime, like a jump from regime 1 to 3,1 to 4 , etc.
} 
the format we discuss by setting $c(t)=a_{i} k(t)-\frac{\dot{k}(t)}{q_{i}}, q_{1}=q(0)$, $q_{2}=q\left(t_{1}\right)$, and accordingly, $V^{i}\left(k(t), \dot{k}(t), t, t_{1}\right)=\ln \left(a_{i} k(t)-\right.$ $\left.\frac{\dot{k}(t)}{q_{i}}\right), i=1,2$. So the constraint functions become $-\dot{k}(t)+$ $q_{i} a_{i} k(t) \geq 0$ and $\dot{k}(t) \geq 0$, for $i=1,2$. Recall that $V$ should be read as $V^{1}$ whenever $t<t_{1}$ and $V^{2}$ whenever $t>t_{1}$.

The planning horizon is infinite. The production function in the consumption sector is simply $a k$, where $a>0$, is the marginal productivity of capital. The consumption good is either used for consumption or as an input in the production of the capital goods. $q(t)$ denotes the linearly expanding technology frontier in the capital goods sector, i.e. $q(t)=1+\gamma t$ measures the productivity in the capital goods sector, and as such, it represents the embodied technical progress variable. We assume without any loss of generality that the capital depreciation rate is nil. We also assume $a_{2}, a_{1}>\rho$, so that the uniformity requirements of our assumptions are verified for the paths of $c(t)$ and $\dot{k}(t)$.

Problem is composed of two phases, where each one corresponds to a different mode of technology. $t_{1}$ refers to the instant of the switching between these modes. At any $t_{1}$, the economy may switch to a more efficient capital goods sector so that the adopted level of technology will be $q\left(t_{1}\right)=1+\gamma t_{1}$, while before switching it is $q(0)=1$. Such a rise in $q$ will only affect the new capital goods, in contrast to an increase in $a$, which is meant to have the same effect on all capital goods whatever the date of their production, whatever their vintage. In this sense, $a$ is neutral and $q$ is investment specific (see Boucekkine et al., 2004). A reassignment of resources towards capital goods due to an increase in $q$ will induce a drop in consumption, thereby resulting with a loss in welfare. This is referred to as obsolescence cost inherent to technology adoption problems (see Boucekkine et al., 2003). In addition to this, switching to a more efficient capital goods sector incurs a loss of technology specific expertise, which can be reflected by $a_{2}<a_{1}$ (see Parente, 1994; Greenwood and Jovanovic, 2001). Given these costs, the trade-off at the basis of the technology adoption problem should be clear by now.

Note by Proposition 3 that $c(t)$ and $k(t)$ are differentiable on each regime. Having this in mind, by the Euler-Lagrange equation (1) for the second regime, we obtain

$k(t)=-A \alpha \mathrm{e}^{a_{2} \alpha t}\left[-\frac{\mathrm{e}^{-\rho t}}{\rho}-\frac{k\left(t_{1}\right) \mathrm{e}^{-a_{2} \alpha t_{1}}}{A \alpha}+\frac{\mathrm{e}^{-\rho t_{1}}}{\rho}\right]$,

where $A=c\left(t_{1}\right) \mathrm{e}^{\left(\rho-a_{2} \alpha\right) t_{1}}, \alpha=1+\gamma t_{1}$. Following from Boucekkine et al. (2004) and Le Van et al. (2007), the necessary transversality condition writes as $\lim _{t \rightarrow \infty}\left(\frac{\partial V}{\partial \dot{k}} k(t) \mathrm{e}^{-\rho t}\right)=0$. Thus, utilizing the Euler-Lagrange equation (1) now for the first period, we find that

$c(t)=c(0) \mathrm{e}^{\left(a_{1}-\rho\right) t}$,

$k(t)=-c(0) \mathrm{e}^{a_{1} t}\left[-\frac{\mathrm{e}^{-\rho t}}{\rho}+\frac{1}{\rho}-\frac{k(0)}{c(0)}\right]$.

Corollary 1 states that $\frac{\partial V}{\partial \dot{k}}$ is continuous at $t_{1}$. Then, from the equality of $\left.\frac{\partial V^{2}}{\partial \dot{k}}\right|_{t_{1}}=\frac{-1}{\rho k\left(t_{1}\right)}$ and $\left.\frac{\partial V^{1}}{\partial \dot{k}}\right|_{t_{1}}=\left.\frac{\partial V^{2}}{\partial \dot{k}}\right|_{t_{1}}$, one can easily find that $c(0)=\rho k\left(t_{1}\right) \mathrm{e}^{\left(\rho-a_{1}\right) t_{1}}$. We also have the continuity of $k(t)$ at $t_{1}$. Evaluating $(13)$ at $t_{1}$, we obtain $k\left(t_{1}\right)=k(0) \mathrm{e}^{\left(a_{1}-\rho\right) t_{1}}$. So we have the solution of the problem in terms of $k(0)$, and $t_{1}$, summarized as follows:

$k(t)=k_{0} \mathrm{e}^{\left(a_{1}-\rho\right) t}, \quad 0<t \leq t_{1}$,
$c(t)=\rho k_{0} \mathrm{e}^{\left(a_{1}-\rho\right) t}, \quad 0<t \leq t_{1}$,
$k(t)=k_{0} \mathrm{e}^{\left(a_{1}-a_{2} \alpha\right) t_{1}} \mathrm{e}^{\left(a_{2} \alpha-\rho\right) t}, \quad t_{1}<t<\infty$,
$c(t)=\frac{\rho}{\alpha} k_{0} \mathrm{e}^{\left(a_{1}-a_{2} \alpha\right) t_{1}} \mathrm{e}^{\left(a_{2} \alpha-\rho\right) t}, \quad t_{1}<t<\infty$.
This solution satisfies the uniformity and the continuity requirements made in the assumptions. In order to proceed to the characterization of the switching instant it only remains to verify Assumption 4 . We need to check only the second period as $t_{1}$ do not occur in the first period solution. For the second regime, $V_{t_{1}}$ is $a_{2} \frac{\mathrm{d} \alpha}{\mathrm{d} t_{1}} t \mathrm{e}^{-\rho t}$, and this is integrable, so that Assumption 4 is satisfied.

Given these, we can proceed to characterize the optimal switching instant by means of the matching condition. We have

$$
\begin{aligned}
& {\left[\dot{x} V_{\dot{x}}^{1}-V^{1}\right]_{t_{1}} \mathrm{e}^{-\rho t_{1}}=-\frac{\left(\rho\left(-1+\ln \left(k_{0} \rho \mathrm{e}^{t_{1}\left(-\rho+a_{1}\right)}\right)\right)+a_{1}\right) \mathrm{e}^{-\rho t_{1}}}{\rho},} \\
& {\left[\dot{x} V_{\dot{x}}^{2}-V^{2}\right]_{t_{1}} \mathrm{e}^{-\rho t_{1}}} \\
& \quad=\frac{\left(\rho-\rho \ln \left(\frac{k_{0} \rho \mathrm{e}^{t_{1}\left(-\rho+a_{1}\right)}}{1+\gamma t_{1}}\right)-a_{2}\left(1+\gamma t_{1}\right)\right) \mathrm{e}^{-\rho t_{1}}}{\rho}, \\
& \int_{t_{1}}^{t_{f}} V_{t_{1}}^{2} \mathrm{e}^{-\rho t} \mathrm{~d} t \\
& =\frac{\left(-\gamma \rho+\left(1+\gamma t_{1}\right)\left(\rho a_{1}-a_{2}\left(\rho+\gamma\left(-1+\rho t_{1}\right)\right)\right)\right) \mathrm{e}^{-\rho t_{1}}}{\left(1+\gamma t_{1}\right) \rho^{2}},
\end{aligned}
$$

and $\int_{0}^{t_{1}} V_{t_{1}}^{1} \mathrm{e}^{-\rho t} \mathrm{~d} t=0$, so the necessary condition for an interior switching turns out to be

$$
\begin{aligned}
& \rho\left[\gamma-\left(1+\gamma t_{1}\right) \rho \ln \left(1+\gamma t_{1}\right)\right] \\
& \quad+\left(1+\gamma t_{1}\right)\left[-2 \rho a_{1}+a_{2}\left(2 \rho+\gamma\left(2 \rho t_{1}-1\right)\right)\right]=0 .
\end{aligned}
$$

After some algebra, and defining $s=1+\gamma t_{1}$, the condition can be recast as

$\rho \gamma+2 \rho a_{2} s^{2}=\rho^{2} s \ln s+s\left(2 \rho\left(a_{1}-a_{2}\right)+a_{2} \gamma+2 \rho a_{2}\right)$.

To simplify the interpretation of (19), we will assume that

$\rho \gamma<2 \rho\left(a_{1}-a_{2}\right)+a_{2} \gamma$.

This condition ensures that the left-hand side of (19) has a lower value than the right-hand side of (19) at $t_{1}=0$. The derivative with respect to $s$ on the left-hand side of (19) is $4 \rho a_{2} s$, while the righthand side derivative is $\rho^{2}(\ln s+1)+2 \rho\left(a_{1}-a_{2}\right)+a_{2} \gamma+2 \rho a_{2}$. Since the derivatives are positive, and for large $s$, the left-hand side derivative will be strictly higher than that of the right-hand side, there exists a unique solution $t_{1}>0$ to (19).

As the matching condition does not have a closed form solution, we shall resort to the numerical analysis and study in particular, the effect of an increase in the growth rate of technology frontier on the optimal timing of technology adoption. We adopt the following set of parameter values: $\rho=0.04, a_{1}=1, a_{2}=0.8$ and $\gamma=0.02$ as our benchmark analysis. We determine that the optimal timing of the switch to the second regime occurs at $t_{1}=25.1$. We obtain that the higher pace of technology implies the fastening of the adoption decision:

\begin{tabular}{|l|l|l|l|}
\hline$\gamma$ & 0.02 & 0.06 & 0.10 \\
\hline$t_{1}$ & 25.10 & 16.64 & 14.98 \\
\hline
\end{tabular}

As a higher technology comes earlier, the loss due to the drop in marginal productivity of capital after adoption becomes tolerable in a shorter run and this also implies that the adopted level of technology to get higher. Similarly, higher discount rates should fasten the adoption. Higher discounting implies an urgency in covering the costs resulting from the delay in adoption.

\begin{tabular}{|l|l|l|l|}
\hline$\rho$ & 0.03 & 0.04 & 0.06 \\
\hline$t_{1}$ & 29.12 & 25.10 & 21.05 \\
\hline
\end{tabular}

In fact the costs from switching decrease at a particular instant with higher discount rates with respect to the costs with a lower discount rates. This is what we see by simply looking at the derivative of (19) with respect to $\rho,-\left(2\left(a_{1}-a_{2}\right)+2 a_{2}\right) s+2 a_{2} s^{2}+\gamma-$ 
$2 s \rho \ln (s)$, as well. On the other hand, the lower value of marginal productivity after adoption delays the adoption:

\begin{tabular}{|l|l|l|l|}
\hline$a_{2}$ & 0.8 & 0.7 & 0.6 \\
\hline$t_{1}$ & 25.10 & 34.25 & 46.50 \\
\hline
\end{tabular}

This is reasonable since lower marginal productivity after adoption means that the cost of switching is higher. So, this should be compensated by a higher gain in technological jump, creating a waiting incentive for a higher technology level to adopt. This is more clear if we consider the derivative with respect to $a_{2}$ of (18), as this derivative, $-\gamma\left(1+\gamma t_{1}\right)+2\left(1+\gamma t_{1}\right)^{2} \rho$, is positive whenever $\rho \geq \frac{\gamma}{2}$.

\subsection{An environmental control problem}

Boucekkine et al. (2010) consider the trade-off between economic performance and environmental quality from the perspective of a government over a finite time horizon by using canonical two-stage optimal control techniques. At any moment in time, the government has to choose when to switch to a new technology which is economically less efficient but better in environmental quality terms. Formally, the environmental control problem that the government endeavor to solve is

$\max _{\left\{C, t_{1}\right\}} \int_{0}^{t_{1}} u(C(t), P(t)) \mathrm{e}^{-\rho t} \mathrm{~d} t+\int_{t_{1}}^{T} u(C(t), P(t)) \mathrm{e}^{-\rho t} \mathrm{~d} t$

subject to the constraints $C(t)+X(t)=F(X(t))=A_{i} X(t), \dot{P}(t)=$ $\alpha_{i} A_{i} X(t)$, with $P(0) \geq 0$, given and $P(T)$ free, where $C, X$, and $P$ denote consumption, input, and pollution, respectively. Given technology $i, A_{i}$ measures the productivity of the input, and $\alpha_{i}$ measures the marginal contribution of an extra unit of production to pollution. The technical menu $\left(A_{1}, \alpha_{1}\right)$ applies on the time span $\left[0, t_{1}\right)$, and the menu $\left(A_{2}, \alpha_{2}\right)$ applies on $\left[t_{1}, T\right]$, where it is assumed that $\alpha_{1}>\alpha_{2}>0$, and $A_{1}>A_{2}>1$.

Considering a utility function of the form $u(C, P)=\ln C-\beta P$, Boucekkine et al. (2010) shows that switching will happen at the corners, unless $\frac{\alpha_{2} A_{2}}{A_{2}-1}=\frac{\alpha_{1} A_{1}}{A_{1}-1}$. In that case, $t_{1}$ can take any value on $[0, T]$ as the government will be indifferent between the two regimes (see Corollary 3 in Boucekkine et al. (2010)). We will now show how this result can easily be obtained with the present approach by utilizing the matching condition (3), without delving into the details of the optimal solution.

The problem can be recast as follows:

$$
\begin{gathered}
\max _{P(t), t_{1}} \int_{0}^{t_{1}} u\left(\frac{\dot{P}(t)}{\alpha_{1} A_{1}}\left(A_{1}-1\right), P(t)\right) \mathrm{e}^{-\rho t} \mathrm{~d} t \\
+\int_{t_{1}}^{T} u\left(\frac{\dot{P}(t)}{\alpha_{2} A_{2}}\left(A_{2}-1\right), P(t)\right) \mathrm{e}^{-\rho t} \mathrm{~d} t
\end{gathered}
$$

subject to $P(t) \geq 0, \dot{P}(t) \geq 0$, with $P(0) \geq 0$, given and $P(T)$ free. Note that, with $P(0)>0$, the optimal solution has to satisfy our Assumption 2 , since $X=0$ derives utility to $-\infty$. That is, the optimal solution satisfies $P(t)>0, \dot{P}(t)>0$ uniformly. The rest of the assumptions are obviously satisfied. In particular, Assumption 4 , has no bite here, as $t_{1}$ does not explicitly appear in the instantaneous utility. Then the interior matching condition writes

$\dot{P} \frac{1}{\dot{P}}-u\left(\frac{\dot{P}}{\alpha_{1} A_{1}}\left(A_{1}-1\right), P\right)=\dot{P} \frac{1}{\dot{P}}-u\left(\frac{\dot{P}}{\alpha_{2} A_{2}}\left(A_{2}-1\right), P\right)$,

which implies

$$
\ln \dot{P}+\ln \left(\frac{A_{1}-1}{\alpha_{1} A_{1}}\right)-\beta P\left(t_{1}\right)=\ln \dot{P}+\ln \left(\frac{A_{2}-1}{\alpha_{2} A_{2}}\right)-\beta P\left(t_{1}\right),
$$

where both sides are evaluated at $t_{1} \cdot u_{\dot{P}}=\frac{1}{\dot{P}}$, is continuous by Corollary 1 , so that the interior matching condition is equivalent to $\frac{A_{1}-1}{\alpha_{1} A_{1}}=\frac{A_{2}-1}{\alpha_{2} A_{2}}$.

Accordingly, the condition for an immediate adoption of the new technology is $\frac{\alpha_{2} A_{2}}{A_{2}-1}<\frac{\alpha_{1} A_{1}}{A_{1}-1}$. Moreover, it is obvious with the present approach that, this result extends easily to the nonlinear pollution disutility and the infinite time horizon cases.

\section{Conclusion}

In this paper, we have analyzed the optimal timing of regime switches in optimal growth models by means of the standard tools of calculus of variations and some basic properties of Sobolev spaces. Our approach has allowed us to consider the three important aspects of the regime switching problems in a simple and unified manner: the infinite planning horizon, multiple regime switches and the explicit dependence of the constraint functions and the objective functional on these switching instants. We have proved that, in addition to the standard optimality conditions such as Euler-Lagrange, two specific sets of necessary conditions that characterize the optimal timing of regime switches emerge: continuity and the matching conditions. We have shown that the Weierstrass-Erdmann corner conditions extend to the problems with regime switches. As for the application, we have considered an optimal adoption problem under embodiment with exogenously growing technology frontier and an environmental control problem with the trade-off between economic performance and environmental quality.

\section{References}

Askenazy, P., Le Van, C., 1999. A model of optimal growth strategy. Journal of Economic Theory 85 (1), 24-51.

Boucekkine, R., del Rio, F., Licandro, O., 2003. Embodied technological change, learning-by-doing and the productivity slowdown. Scandinavian Journal of Economics 105 (1), 87-97.

Boucekkine, R., Krawczyk, J.B., Vallée, T., 2010. Environmental quality versus economic performance: a dynamic game approach. Optimal Control Applications and Methods doi:10.1002/oca.927.

Boucekkine, R., Saglam, C., Vallée, T., 2004. Technology adoption under embodiment: a two-stage optimal control approach. Macroeconomic Dynamics 8, 250-271.

Brezis, H., 1983. Analyse fonctionelle: théorie et applications, in: Ciarlet, P.G., Lions, J.L. (Eds.), Collection mathematiques appliquees pour la maitrise. Masson, Paris, Buttazzo, G., Giaquinta, M., Hildebrandt, S., 1998. One Dimensional Variational Problems, An Introduction. Oxford Science Publications.

Dana, R., Le Van, C., 2003. Variational Calculus in Infinite Horizon. Mimeo, Universite Paris-1, Pantheon-Sorbonne.

Greenwood, J., Jovanovic, B., 2001. Accounting for growth. In: Dean, E., Harper, M., Hulten, C. (Eds.), New Directions in Productivity Analysis. In: NBER Studies in Income and Wealth, vol. 63. Chicago University Press, Chicago.

Lang, S., 1993. Real and Functional Analysis. Springer-Verlag, New York Inc.

Le Van, C., Boucekkine, R., Saglam, C., 2007. Optimal control in infinite horizon problems: a Sobolev space approach. Economic Theory 32 (3), 497-509.

Makris, M., 2001. Necessary conditions for infinite horizon discounted two-stage optimal control problems. Journal of Economic Dynamics and Control 25, 1935-1950.

McKenzie, L.W., 1986. In: Arrow, K., Intriligator, M. (Eds.), Optimal Economic Growth, Turnpike Theorems and Comparative Dynamics. In: Handbook of Mathematical Economics, vol. III. North-Holland, Amsterdam, pp. 1281-1355.

Parente, S., 1994. Technology adoption, learning by doing, and economic growth. Journal of Economic Theory 63, 346-369.

Saglam, C., 2010. Optimal pattern of technology adoptions under embodiment: a multi-stage optimal control approach. Optimal Control Applications and Methods doi:10.1002/oca.960.

Stokey, N.L., Lucas Jr., R.E., 1989. Recursive Methods in Economic Dynamics. Harvard University Press, Cambridge.

Tomiyama, K., 1985. Two-stage optimal control and optimality conditions. Journal of Economic Dynamics and Control 9, 315-337.

Tomiyama, K., Rossana, R.J., 1989. Two-stage optimal control problems with an explicit switch point dependence. Journal of Economic Dynamics and Control $13,319-337$. 